\title{
EVALUACIÓN DE SIMULACIONES DE LA PRECIPITACIÓN EN CLIMA PRESENTE USANDO EL MODELO WRF EN LA PENÍNSULA IBÉRICA
}

\author{
M. GARCÍA-VALDECASAS-OJEDA, S. de FRANCISCIS, S.R. GÁMIZ-FORTIS, \\ Y. CASTRO-DÍEZ and M.J. ESTEBAN-PARRA \\ Departamento de Física Aplicada, Universidad de Granada, España. \\ mgvaldecasas@ugr.es
}

\section{RESUMEN}

En este estudio se ha llevado a cabo un conjunto de simulaciones de alta resolución espacio-temporal para un periodo de 31 años haciendo uso del modelo regional Weather Reserch and Forecasting (WRF) con el fin de evaluar la capacidad del modelo para simular la variabilidad de las precipitaciones en la Península Ibérica. Las simulaciones se realizaron usando como condiciones iniciales y de contorno los datos de reanálisis de ERA-Interim, como "condiciones de contorno perfectas", y las salidas globales del modelo CMIP5 CESM corregidas en sesgo, sobre un dominio centrado en la PI anidado en el dominio EURO-CORDEX. La evaluación se basó en la comparación de las salidas del modelo con los datos observacionales Spain02 para las precipitaciones en España y PT02 para Portugal, a diferentes escalas temporales con el objetivo de poder averiguar si las simulaciones regionalizadas son capaces de capturar tanto los valores medios como los eventos extremos.

Los resultados indican que aunque existen ciertos errores substanciales, WRF es capaz de capturar los principales patrones espaciales de la precipitación en la PI, resultando una herramienta útil a la hora de realizar simulaciones regionales para zonas con una topografía compleja como es la Península Ibérica.

Palabras clave: WRF, downscaling dinámico, Península Ibérica, precipitaciones, simulaciones climáticas regionales, evaluación climática.

\begin{abstract}
A set of 31-yr high-resolution simulations has been carried out with the regional Weather Research and Forecasting (WRF) model in order to evaluate its capability to simulate precipitation variability in the Iberian Peninsula. The WRF model was forced by the ERA-Interim data as "perfect boundary conditions", and the global bias-corrected climate model outputs from CMIP5 CESM model, over a domain encompassing the IP and nested in the coarser EURO-CORDEX domain. Evaluation was based on comparison at different time scales in order to assess the model ability to capture long-term mean precipitation values and high-order statistics (extreme events). For this end, we used two different observational gridded datasets: the Spain02 data for Spanish precipitation and the PT02 data for Portugal.
\end{abstract}

Although considerable errors are still observed, results show that WRF is able to capture the main spatial precipitation patterns in IP. Therefore, we can say that WRF 
provides useful information at regional scale, with significant improvement in complex terrain areas such as Iberian Peninsula.

Key words: WRF, dynamical downscaling, Iberian Peninsula, precipitations, regional climate simulations, climate evaluation.

\section{INTRODUCCIÓN}

La precipitación es sin duda una de las variables climáticas con mayor repercusión en el ser humano, capaz de causar gran impacto en la sociedad y el medio ambiente. Este hecho hace indispensable una correcta caracterización de los patrones de variabilidad de la precipitación así como el estudio de su evolución en un contexto de cambio climático. El uso de herramientas que aporten información adecuada sobre cómo se comportará esta variable es importante a la hora de realizar una gestión adecuada para intentar mitigar los efectos que se producirán en distintos ámbitos tan importantes como la agricultura o el abastecimiento urbano.

Dentro de este contexto, los Modelos Regionales del Clima (RCMs) son una fuente primordial de información en estudios de cambio climático, generando un valor añadido a la hora de representar la variabilidad climática a escala regional. Ahora bien, debido a la existencia de las diferentes fuentes de error que hay en los RCM, es conveniente que para realizar proyecciones del clima futuro se efectúe una evaluación previa del modelo de predicción, ya que una mejor representación en el presente implicará proyecciones en el futuro más fiables. Hay que notar que la representación correcta por parte del modelo en el presente no implica resultados adecuados en el futuro, pero aportará mayor fiabilidad a los resultados. Además, la evaluación del modelo a usar ayudará a analizar las habilidades y debilidades del sistema de predicción así como a corregir posibles sesgos sistemáticos que pudieran detectarse en las salidas de simulaciones en tiempo presente, y en consecuencia aplicarse en proyecciones futuras de cambio climático.

Por ello en este estudio se evalúa la capacidad del modelo Weather Research and Forecasting (WRF) Model para simular las precipitaciones en la Península Ibérica (PI) tomando como condiciones iniciales y de contorno las salidas de un Modelo Climático General (GCM). Dichas salidas son comparadas con las obtenidas con datos de reanálisis. De esta manera, podrán compararse las diferencias que se producen al tomar como condiciones de contorno un GCM con las obtenidas con datos de reanálisis y datos observacionales pudiendo dilucidar si las salidas de WRF para dicho GCM son representativas del clima presente y por tanto adecuadas a la hora de realizar proyecciones de cambio climático a escala regional.

\section{MÉTODOS}

\subsection{Configuración del modelo}

En este estudio se ha utilizado la versión 3.6.1 del modelo WRF-ARW (Skamarok et al., 2008) para la realización de simulaciones regionales de clima presente. El modelo WRF es un modelo de predicción regional ampliamente aceptado por la comunidad científica para su uso en simulaciones de clima presente y proyecciones de clima futuro de alta resolución. 
Cada una de las simulaciones se realizó a una resolución espacial de $0.088^{\circ}$ $(\sim 10 \mathrm{~km})$ sobre un dominio centrado en la PI (D02) anidado sobre el dominio EUROCORDEX (D01) de $0.44^{\circ}$ de resolución $(50 \mathrm{~km})$ horizontal (Fig. 1). En la vertical, ambos dominios se componen de 41 niveles verticales de presión, situándose el nivel más alto en $10 \mathrm{hPa}$. El periodo de estudio utilizado ha sido desde enero de 1979 hasta noviembre de 2010 con un spin-up de 11 meses. Como condiciones iniciales y de contorno se han usado dos fuentes de datos diferentes: los datos de reanálisis de ERA-Interim (Berrisford et al., 2011) como "condiciones de contorno ideales" (cuyas simulaciones serán denominadas como WRFERA) y los datos con corrección de sesgos de las salidas del modelo global CMIP5 CESM1 (Bruyère et al., 2014) (sus salidas serán denominadas WRFCCSM4).

Con el objetivo de evitar inconsistencias en los bordes del dominio se aplicó una técnica de nudging espectral (von Storch et al., 2000) sobre todas las variables de entrada excepto para la humedad. Esta técnica se aplicó sobre el dominio más grande y por encima de la capa límite, permitiendo así que el modelo genere su propia dinámica interna para el dominio anidado.

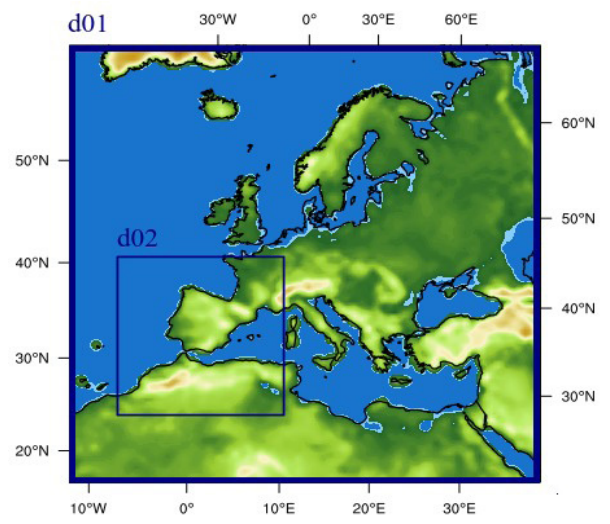

Fig. 1: Dominio de estudio.

El conjunto de parametrizaciones utilizadas son: Asymmetric convective model version 2 (ACM2; Pleim, 2007) para capa límite; Betts-Miller-Janjic (BMJ; Betts and Miller, 1986; Janjic, 1990,1994) como modelo de convección; WRF single-moments three class schemes (WRF3S; Hong et al., 2004) para microfísica de nubes; Noah LSM (Chen and Dudhia, 2001) como modelo de suelo; y Community Atmosphere Model 3.0 (CAM3.0; Collins et al., 2004) para radiación de onda corta y radiación de larga.

\subsection{Evaluación de las precipitaciones}

La evaluación del modelo WRF se ha realizado a diferentes escalas temporales (desde anual a diaria) con el objetivo de comprobar si el modelo representa de manera adecuada la variabilidad del clima presente en la PI. Para ello, las salidas de precipitación de WRF se han comparado con los datos observacionales de Spain02 (versión 4) y PT02 (versión 1) para España peninsular e Islas Baleares y Portugal, respectiva- 
mente. Spain02 es una base de datos en rejilla de precipitaciones observadas a escala diaria desarrollada por Herrera et al. (2016) a partir de registros de estaciones de la Agencia Estatal de Meteorología Española de alta calidad. Dentro de las diferentes resoluciones disponibles para la versión 4, en este estudio se han utilizado los datos en rejilla a resolución horizontal de $0.11^{\circ}$ con coordenadas rotadas según los criterios de EURO-CORDEX, disponiendo de registros comprendidos entre 1971 y 2010. Por su parte, la base de datos observacionales PT02 desarrollada por Belo-Pereira et al., (2011) contiene las series de precipitación diaria de Portugal en una rejilla regular de $0.2^{\circ}$ de resolución horizontal para el periodo comprendido entre 1950 y 2003.

Debido a que la resolución horizontal de cada base de datos es diferente, el primer paso realizado fue el regrillado, mediante interpolación lineal, de las salidas del modelo WRF a la resolución de las bases de datos observacionales en cada caso con el fin de hacer los datos comparables entre ellos. De esta forma, la evaluación se ha realizado punto a punto de forma independiente para España y Portugal.

\section{RESULTADOS}

\subsection{Precipitaciones acumuladas anuales}

Las precipitaciones acumuladas anuales han sido calculadas para los datos observados y simulados obteniéndose posteriormente el promedio para el periodo completo de estudio en cada caso y para cada punto de rejilla. Dicho periodo fue 1979-2010 para España y 1979-2003 para Portugal. El objetivo de la representación del valor medio acumulado para todo el periodo de estudio es analizar si el modelo es capaz de representar los patrones principales de variabilidad de la precipitación en la PI e identificar las zonas que presentan mayor desviación con respecto a los valores observados. La Fig. 2 muestra la precipitación acumulada anual media obtenida a partir de los datos de Spain02 y PT02 (representándose en el mismo mapa, por lo que se le nombrará a partir de ahora como SPAINPT02), y de las simulaciones WRFERA y WRFCCSM4. Con el propósito de cuantificar el grado de similitud de los patrones espaciales obtenidos se han calculado los coeficientes de correlación de Pearson de los patrones simulados con respecto a SPAINPT02 (mostrados entre paréntesis). Como puede verse, de manera general, WRF representa adecuadamente la media climática anual para la PI, simulando los patrones principales de variabilidad espacial al identificar el gradiente noroeste-sureste de precipitaciones que caracteriza al clima de esta zona. Dicho gradiente queda bien representado en ambas simulaciones, WRFERA y WRFCCSM4, siendo los resultados entre ambas simulaciones muy similares, aunque ligeramente mejores en el caso de WRFERA. Respecto a la correlación espacial, puede verse que ambas simulaciones presentan altos valores de correlación (0.89 y 0.87 para WRFERA y WRFCCSM4, respectivamente) siendo ligeramente mejor para las salidas que utilizan como condiciones de contorno los datos de ERA-Interim. Por otro lado, aunque de manera general WRF represente bien la media climática, existen errores que dan lugar a subestimaciones en algunos casos (Cuenca del río Ebro, sur y sureste peninsular y Galicia), y sobreestimaciones en otros, como por ejemplo, en el Sistema Ibérico y sobre todo en la Cordillera Cantábrica, donde WRF simula precipitaciones superiores a $2600 \mathrm{~mm} /$ año mientras que los datos observacionales indican precipitaciones en torno a $1600 \mathrm{~mm} /$ anuales. 

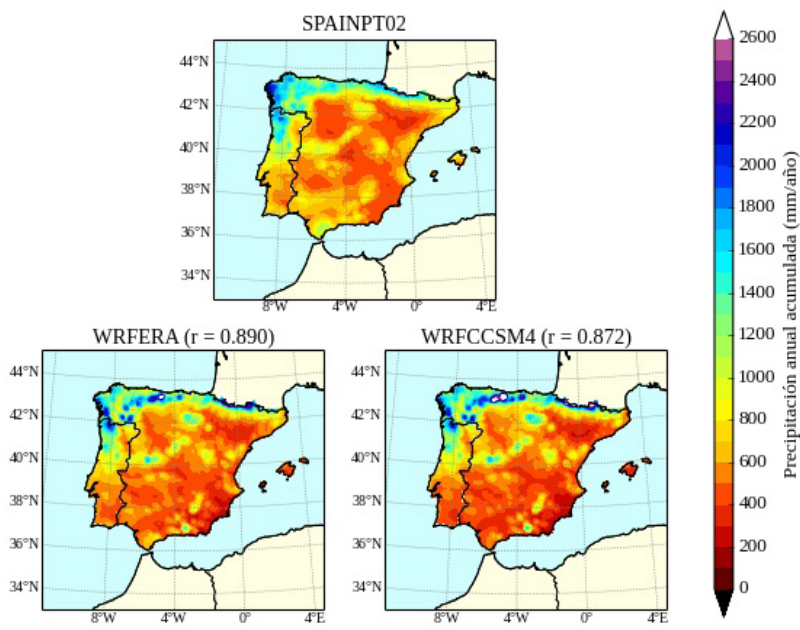

Fig. 2: Precipitación acumulada anual media obtenida de SPAINPT02, WRFERA y WRFCCSM4. En paréntesis se muestra el coeficiente de correlación espacial con respecto a las observaciones.

\subsection{Sesgos anuales y estacionales de las precipitaciones acumuladas}

Otra medida de la idoneidad del modelo para la simulación de las precipitaciones en la PI, es el sesgo (BIAS) relativo entre las precipitaciones simuladas y observadas a escala anual y estacional, tomando como invierno los meses entre diciembre y febrero (DEF), primavera el periodo de marzo a mayo (MAM), verano de junio a agosto (JJA) y otoño de septiembre a noviembre (SON). Para tal efecto, se entiende como sesgo al sumatorio de la diferencia entre los datos observados y simulados de las series temporales. Con el fin de comparar diferentes regiones, estas diferencias se dividen entre el valor promedio de las observaciones para todo el periodo de estudio, obteniéndose el BIAS relativo en tanto por ciento. Como puede verse en la Fig. 3, WRFERA presenta sesgos moderados a escala anual, obteniéndose valores de BIAS comprendidos entre 25 y $-25 \%$ en la mayor parte de la PI e Islas Baleares. Excepciones de ello son el sur de la Península y la Costa Mediterránea con subestimaciones de hasta un 50\% de las precipitaciones acumuladas a escala anual. El aumento del sesgo en estas regiones se debe, en cierta medida, a que las precipitaciones en estas zonas son menores, siendo el sesgo relativo superior para errores similares a los ocurridos en la zona norte o centro donde la precipitación es mayor. En contraposición, y como puede verse en la Fig. 2 el modelo sobrestima las precipitaciones del centro-norte peninsular coincidiendo con regiones montañosas como la zona de los Pirineos. Resultados similares se muestran para las salidas de WRFCCSM4, siendo los errores de subestimación y sobreestimación más generalizados y/o acusados.

Estacionalmente, se muestran valores mayores de sesgo, tanto de signo positivo como negativo, en comparación con los mostrados a escala anual que probablemente pueden estar compensados. Dichos sesgos son más marcados en verano (JJA) y otoño (SON) donde pueden verse valores de BIAS negativo en el sur peninsular superiores al $100 \%$ en algunos casos. 

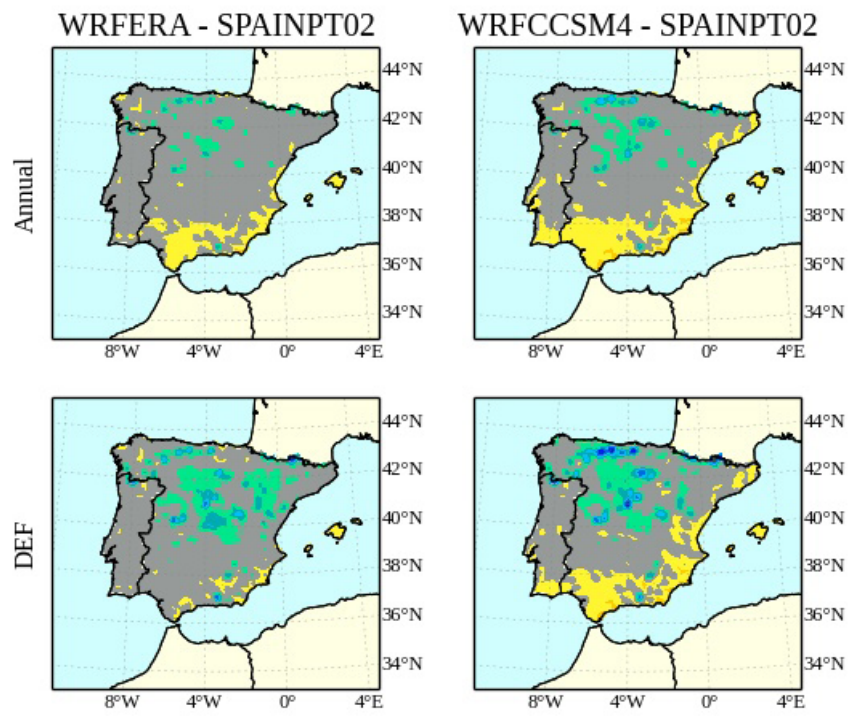

150

125
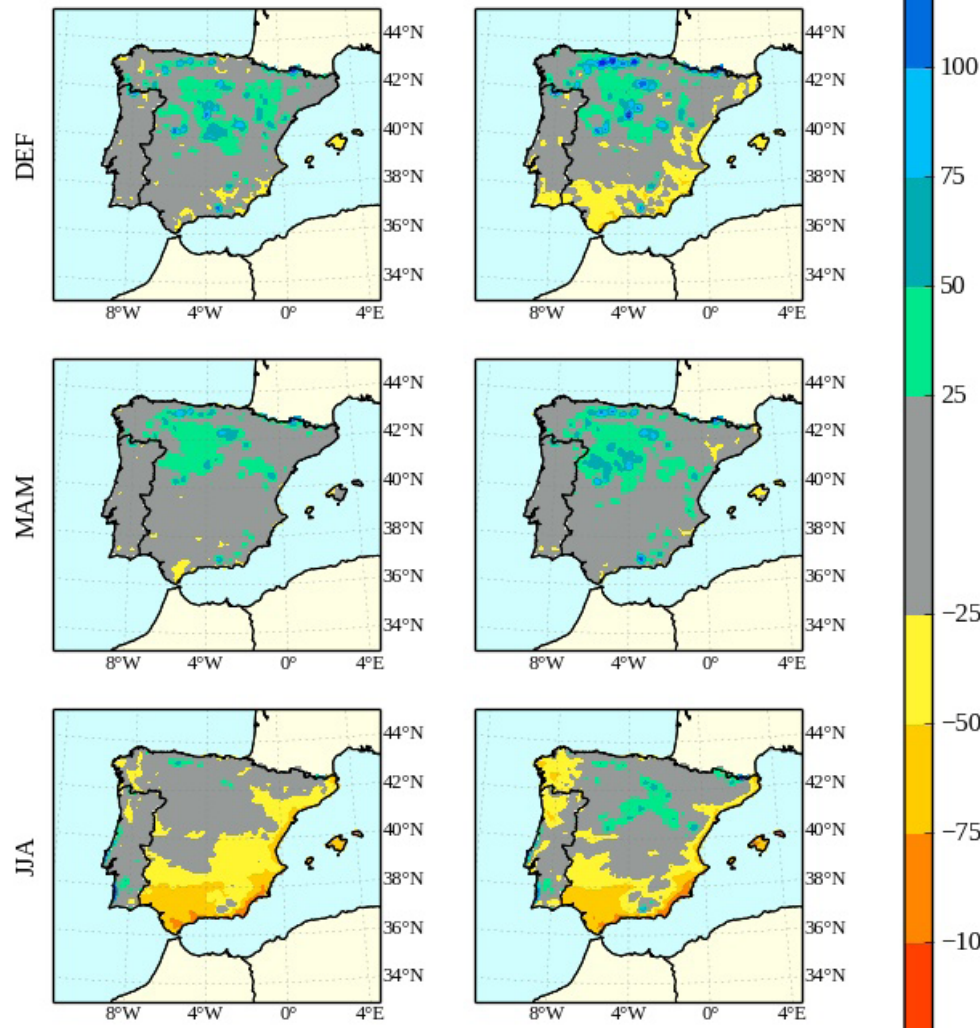

$-50$

ఏ
0
0
0
0
0
0
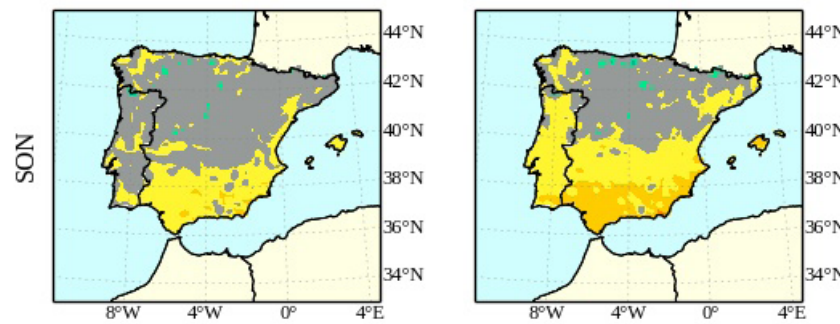

$-125$

Fig. 3: Sesgo relativo (\%) de las precipitaciones anuales y estacionales acumuladas simuladas por WRFERA y WRFCCSM4 con respecto a los datos observacionales SPAINPT02. 
En cuanto a las diferencias entre las distintas condiciones iniciales y de contorno, los resultados muestran un cierto grado de similitud en muchos casos, presentándose valores de sesgos positivo en el centro y norte peninsular así como valores negativos para la zona sur y Galicia. Las diferencias más notables entre los datos de WRFERA y WRFCCSM4 son una subestimación más acusada para los datos de WRFCCSM4 en invierno, verano y otoño en el sur, la existencia de sesgos positivos en verano para el centro peninsular o los valores de precipitación más acusados en la zona cantábrica para invierno en los datos de WRFCCSM4.

\subsection{Análisis de precipitaciones diarias: valores extremos}

La Fig. 4 muestra la similitud, en tanto por ciento, entre la función de distribución de probabilidad (PDF) de las precipitaciones acumuladas diarias simuladas con respecto a las observadas a través del denominado Skill Score (SS; Perkins et al., 2007). El SS compara las PDFs de las precipitaciones diarias simuladas y observadas para lo cual toma como medida de similitud el área común bajo estas dos funciones, obtenidas utilizando como intervalo de estudio $1 \mathrm{~mm}$. Así, el valor de $100 \%$ indica que ambas funciones de distribución son iguales mientras que el $0 \%$ significa que no tienen nada en común.

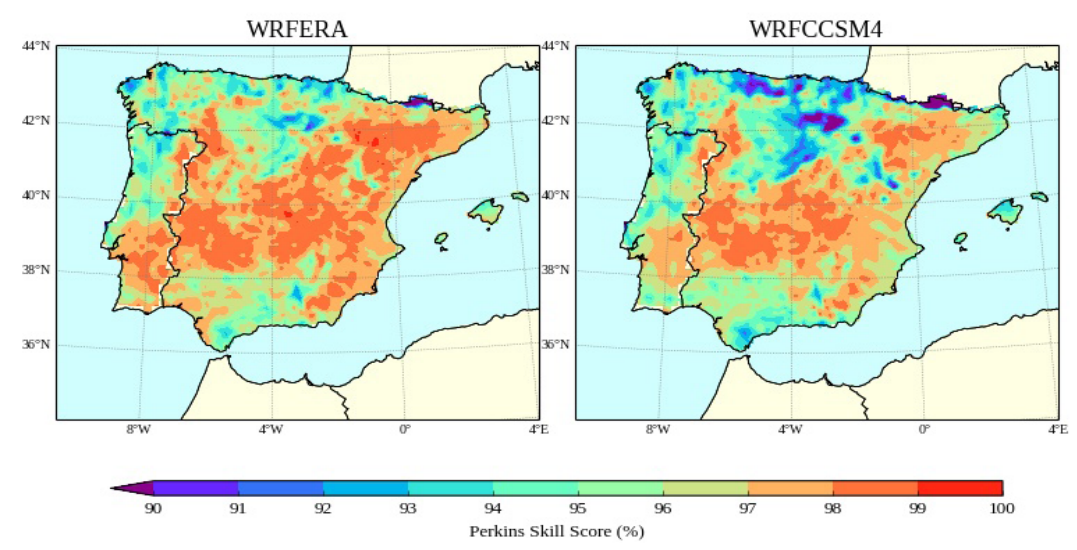

Fig. 4: Perkins Skill Score (\%) de las precipitaciones diarias acumuladas simuladas por WRFERA y WRFCCSM4 con respecto a los datos observacionales SPAINPT02.

En la mayoría de los casos, los valores de SS se encuentran comprendidos entre el 90 y 100\%, siendo esta medida de similitud más baja para WRFCCSM4, en general para toda la PI. Las PDFs obtenidas a partir de los valores simulados y observados en distintas zonas del norte peninsular parecen mostrar mayores diferencias. En contraposición, WRF muestra una mayor habilidad para capturar las precipitaciones diarias en la cuenca del Ebro, así como en el sureste, suroeste y centro peninsular.

Por último, y con el objetivo de evaluar la simulación de valores extremos de precipitación en cuanto a su intensidad, se han calculado diferentes índices extremos sugeridos por el ETCCDI (Expert Team on Climate Change Detection and Indices). Los índices analizados se encuentran recogidos en la Tabla 1. 


\begin{tabular}{|l|l|l|}
\hline Índice & Descripción & Unidades \\
\hline R10 & Días con precipitaciones fuertes $(>10 \mathrm{~mm})$. & días/año \\
\hline R20 & Días con precipitaciones muy fuertes $(>20 \mathrm{~mm})$. & días/año \\
\hline CDD & Máximo número de días con precipitaciones $<1 \mathrm{~mm}$. & días/año \\
\hline CWD & Máximo número de días con precipitaciones $>=1 \mathrm{~mm}$. & días/año \\
\hline RX5day & Máximas precipitaciones en 5 días consecutivos. & $\mathrm{mm} / \mathrm{año}$ \\
\hline SDII & Intensidad media para días con precipitaciones $>=1 \mathrm{~mm}$. & $\mathrm{mm} / \mathrm{año}$ \\
\hline R95pToT & $\begin{array}{l}\text { Porcentaje de precipitación de eventos de precipitaciones superiores al } \\
\text { percentil 95. }\end{array}$ & $\%$ \\
\hline
\end{tabular}

Tabla 1: índices extremos sugeridos por el ETCCDI calculados en este estudio.

Para el cálculo de estos índices se ha hecho uso del software MeteoLab, desarrollado por el Grupo de Meteorología de Santander. Así, se han calculado cada uno de los índices para cada año, obteniéndose finalmente la media anual para todo el periodo de estudio (diciembre-1979 a noviembre-2010 para España, y diciembre-1979 a noviembre-2003 para Portugal).

La Fig. 5 presenta el valor promedio de cada índice en el periodo 1979-2010 para España y 1979-2003 para Portugal, calculados a partir de los datos observacionales, las salidas de WRFERA y de WRFCCSM4. El modelo WRF representa de manera adecuada las precipitaciones en la PI en términos de índices extremos, presentando pocas diferencias entre los resultados obtenidos con WRFERA y WRFCCSM4. En cuanto a los resultados de R10 y R20 puede verse cómo WRF subestima el número de días con precipitaciones superiores a 10 y $20 \mathrm{~mm}$ en toda la región sur de la península, siendo dicha subestimación más acusada en WRFCCSM4. Este mismo comportamiento se encuentra en parte de la región gallega. En contraposición, WRF sobreestima el número de días con precipitaciones fuertes o muy fuertes en zonas de la Cordillera Cantábrica.

Respecto a los índices relacionados con el máximo número de días consecutivos con precipitaciones inferiores a $1 \mathrm{~mm}(\mathrm{CDD})$ se puede resaltar que WRF, para ambas condiciones iniciales y de contorno, indica un valor medio más elevado en el sur, en especial para Cádiz. A partir del índice RX5day, el cual indica las precipitaciones máximas en 5 días consecutivos, puede verse cómo WRF sobreestima las precipitaciones en la Cordillera Cantábrica y en el Sistema Central, siendo más visible la sobreestimación en las simulaciones de WRFCCSM4. En el caso del índice SDII, o intensidad media, se observa una mayor diferencia entre los datos WRFERA y WRFCCSM4 dando lugar este último a una subestimación generalizada para toda la zona central de España y Portugal.

\section{DISCUSIÓN}

En este estudio se ha evaluado la capacidad del modelo WRF para representar el comportamiento de las precipitaciones en la PI a distintas escalas temporales, con el objetivo de analizar si el modelo es capaz de reproducir tanto los valores medios climáticos como los eventos extremos. Para ello, se comparan las salidas de precipitación de WRF con los datos observacionales en rejilla de Spain02 y PT02 de España y Portugal, respectivamente. 

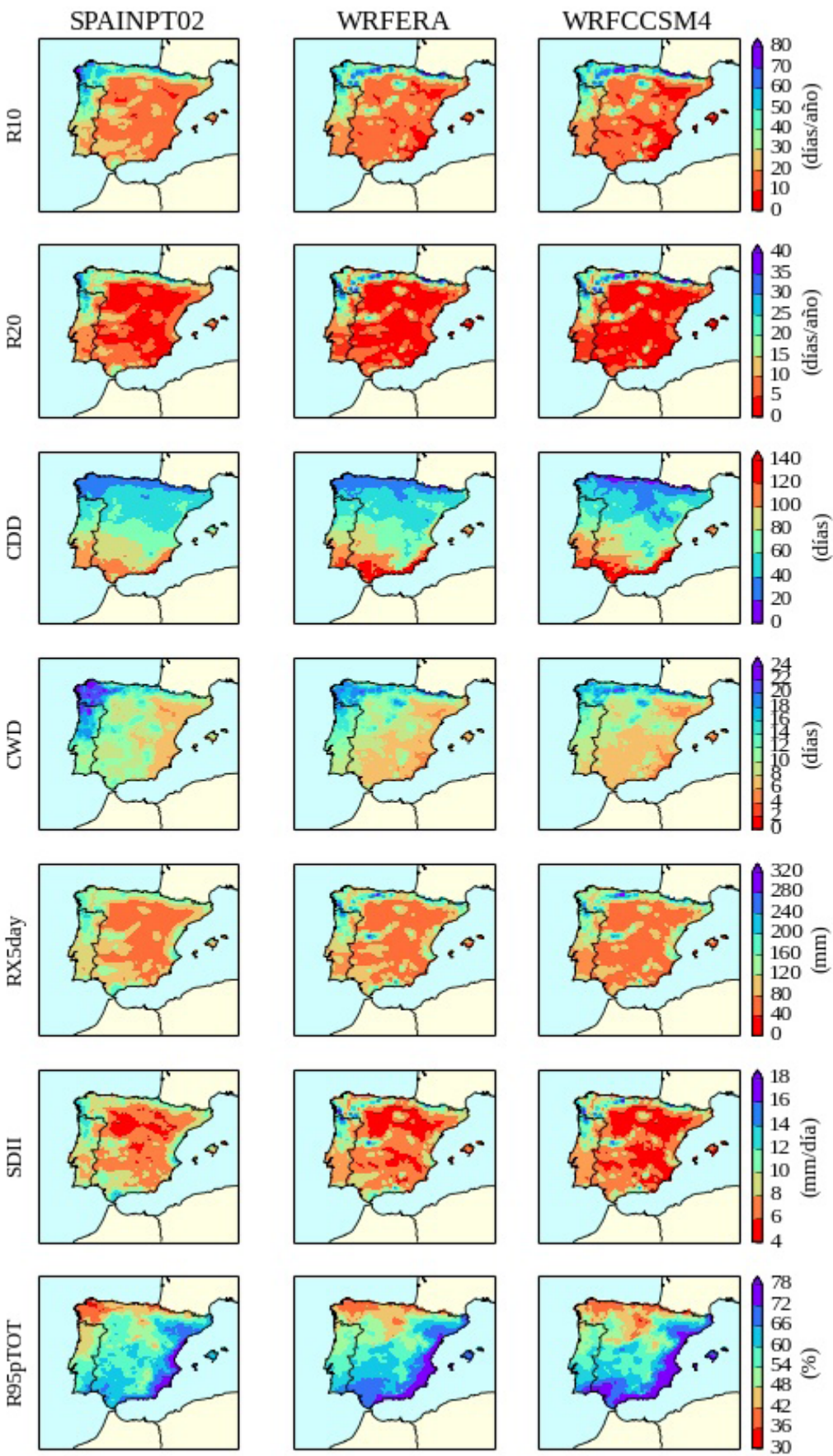

Fig. 5: Índices extremos propuestos por el ETCCDI calculados a partir de los datos observacionales SPAINPT02 y de las simulaciones WRFERA y WRFCCSM4. 
Siendo el objetivo final evaluar la idoneidad del modelo WRF para realizar proyecciones regionales de cambio climático a partir de las salidas del GCM CMIP5 CESM1, se utilizaron dos bases de datos como condiciones iniciales y de contorno: los datos de reanálisis de ERA-Interim como "condiciones de contorno perfectas" y las salidas corregidas en sesgo del modelo global CMIP5 CESM1. En general, los resultados sugieren que aunque aún existen errores importantes en la simulación de la precipitación anual para España y Portugal, WRF simula de una manera adecuada los patrones espaciales de la misma, siendo por tanto una herramienta muy útil para representar la precipitación de la PI a escala regional. Tras la comparación de los resultados con las diferentes salidas de WRF, se encuentra que el uso de las salidas corregidas en sesgo del GCM CMIP5 CESM1, como condiciones iniciales y de contorno, parece ser adecuado para realizar proyecciones de cambio climático a alta resolución espacial y temporal en un terreno complejo como la PI, al menos en lo que a la precipitación se refiere.

\section{AGRADECIMIENTOS}

Este estudio se realizó usando los datos de Spain02 v4 proporcionados por el grupo de Meteorología de Santander, y los datos de PT02 proporcionados por el Instituto de Meteorología de Portugal y las salidas del modelo global CMIP5 CESM1 (http:// rda.ucar.edu). El software libre de MeteoLab está disponible en http://www.meteo. unican.es. Las salidas climáticas del modelo WRF se realizaron en el servicio de computación ALHAMBRA de la Universidad de Granada. Este estudio está financiado por los proyectos P11-RNM-7941 (Junta de Andalucía-Spain) y CGL2013-48539-R (MINECO-Spain, FEDER).

\section{REFERENCIAS}

Belo-Pereira, M., Dutra, E. and Viterbo, P. (2011). Evaluation of global precipitation data sets over the Iberian Peninsula. Journal of Geophysical Research 116, D20101, doi:10.1029/2010JD015481

Berrisford, P., Kållberg, P., Kobayashi, S., Dee, D., Uppala, S., Simmons, A.J., Poli, P. and Sato, H., (2011). Atmospheric conservation properties in ERA-Interim. Quarterly Journal of the Royal Meteorological Society 137, 1381-1399, doi:10.1002/qj.864

Betts, A.K. and Miller, M.J. (1986). A new convective adjustment scheme. Part II: Single column tests using GATE wave, BOMEX, ATEX and arctic air-mass data sets. Quarterly Journal of the Royal Meteorological Society , 112, 693-709, doi:10.1002/qi.49711247308

Bruyère, C.L., Done, J.M., Holland, G.J. and Frederik, S., (2014). Bias corrections of global models for regional climate simulations of high-impact weather. Climate Dynamics 43, 1847-1856, doi:10.1007/s00382-013-2011-6

Chen, F. and Dudhia, J. (2001). Coupling an Advanced Land Surface-Hydrology Model with the Penn State-NCAR MM5 Modeling System. Part I: Model Implementation and Sensitivity. Monthly Weather Review, 129, 569-58, doi:10.1175/1520-0493(2001)

Collins, W.D., et al. (2004). Description of the NCAR community atmosphere model (CAM 3.0), NCAR technique note/TN-464+STR, doi:10.5065/D63N21CH 
Hong, S.Y., Lim, K.S.S., Kim, J.H., Lim, J.O.J. and Dudhia J. (2009). Sensitivity study of cloud-resolving convective simulations with WRF using two bulk microphysical parameterizations: Ice-phase microphysics versus sedimentation effects. Journal of Applied Meteorology and Climatology, 48, 61-76, doi:10.1175/2008JAMC1960.1

Herrera, S., Fernández, J., and Gutiérrez, J.M. (2016). Update of the Spain02 Gridded Observational Dataset for Euro-CORDEX evaluation: Assessing the Effect of the Interpolation Methodology. International Journal of Climatology 36, 900-908, doi: $10.1002 /$ joc. 4391

Janjic, Z.I. (1990). The step-mountain coordinate. Physical package, Monthly Weather Review, 118, 1429-1443, doi:10.1175/1520-0493(1990)118<1429:TSMCPP>2. $0 . \mathrm{CO} ; 2$.

Janjic, Z.I. (1994). The step-mountain eta coordinate model: Further developments of the convection, viscous sublayer, and turbulence closure schemes, Monthly Weather Review,122, 927-945, doi:10.1175/1520-0493(1994)122,0927:TSMEC M.2.0.CO;2.

Perkins, S.E., Pitman, A.J., Holbrook, N.J. y McAneney, J. (2007). Evaluation of the AR4 climate models simulated daily maximum temperature, minimum temperature, and precipitation over Australia using probability density functions. Journal of Climatology 20, 4356-4376, doi:10.1175/JCLI4253.1

Pleim, J.E. (2007). A combined local and nonlocal closure model for the atmospheric boundary layer. Part I: Model description and testing, Journal of Applied. Meteorology and Climatology, 46, 1383-1395, doi:10.1175/JAM2539.1

Skamarock, W.C., Klemp, J.B., Dudhia, J., Gill, D.O., Barker, D.M., Dudha, M.G., Huang, X., Wang, W. and Powers, Y. (2008). A description of the advanced research WRF Ver. 30. NCAR technical note (p. 113). NCAR/TN-475. Disponible on-line en: www.mmm.ucar.edu/wrf/users/docs/arw_v3.pdf

von Storch, H., Langenberg, H. and Feser, F. (2000). A spectral nudging technique for dynamical downscaling purposes. Monthly Weather Review 128, 3664-3673, doi:10.1175/15200493(2000)128<3664:ASNTFD>2.0.CO;2 